\title{
Convergence and Stability of the Truncated Euler- Maruyama Method for Stochastic Differential Equations with Piecewise Continuous Arguments
}

\author{
Yidan Geng ${ }^{1}$, Minghui Song ${ }^{1, *}$, Yulan $\mathrm{Lu}^{2}$ and Mingzhu Liu ${ }^{1}$ \\ ${ }^{1}$ Department of Mathematics, Harbin Institute of Technology, Harbin \\ 150001, China \\ ${ }^{2}$ Institute of Computational Mathematics and Scientific/Engineering \\ Computing, Academy of Mathematics and Systems Science, Chinese \\ Academy of Sciences, Beijing 100190, China
}

Received 21 July 2019; Accepted (in revised version) 4 July 2020

\begin{abstract}
In this paper, we develop the truncated Euler-Maruyama (EM) method for stochastic differential equations with piecewise continuous arguments (SDEPCAs), and consider the strong convergence theory under the local Lipschitz condition plus the Khasminskii-type condition. The order of convergence is obtained. Moreover, we show that the truncated EM method can preserve the exponential mean square stability of SDEPCAs. Numerical examples are provided to support our conclusions.
\end{abstract}

AMS subject classifications: $65 \mathrm{C} 30$

Key words: Stochastic differential equations with piecewise continuous argument, local Lipschitz condition, Khasminskii-type condition, truncated Euler-Maruyama method, convergence and stability.

\section{Introduction}

Differential equations with piecewise continuous arguments (EPCAs) have the following form

$$
d x(t)=a(x(t), x(u(t))) d t
$$

where the argument $u(t)$ has the intervals of constancy, such as $u(t)=[t],[t-n], t-n[t]$. EPCAs have plenty of useful applications in the stabilization of hybrid control systems with feedback discrete controller $[9,17,25,28]$. Note that $[t]$ is a discontinuous function and the solutions are determined by a finite set of initial data, rather than an initial function. Moreover, EPCAs represent a hybrid of continuous and discrete dynamical systems and combine the properties of both differential and difference equations [27].

\footnotetext{
${ }^{*}$ Corresponding author. Email addresses: ydgeng@hit.edu.cn (Y. Geng), luyulan2013@163.com (Y. Lu), songmh@lsec.cc.ac.cn (M. Song), mzliu@hit.edu.cn (M. Liu)
} 
However, [21] pointed out that substantial theories of functional differential equations did not exist for differential equations with piecewise constant or piecewise continuous arguments. Therefore, it is necessary to extend the theory of delay differential or functional differential equations with continuous arguments to equations with discontinuous arguments.

Actually, the effects of noise are not negligible for some systems [22]. Therefore, the stochastic differential equations with piecewise continuous arguments (SDEPCAs) are used to describe many practical problems. It has been widely used in the neural networks, control theory and so on $[11,23,28]$. Since there is, in general, no explicit solutions to SDEPCAs, numerical solutions are required in practice. The convergence and stability of numerical methods are the central properties in numerical analysis, and it is well studied for SDEPCAs under global Lipschitz and linear growth conditions, such as [20]. However, many systems do not satisfy the global Lipschitz or the linear growth conditions. Song et al. [24] investigated the convergence of the tamed Euler method for SDEPCAs under non-global Lipschitz continuous coefficients.

According to [7], the classical explicit Euler method diverges when the coefficients of stochastic differential equations (SDEs) are super-linearly growing. To overcome this difficulty, some scholars turn to use implicit Euler methods, such as the semiimplicit Euler-Maruyama method, the backward Euler-Maruyama method and the splitstep theta (SST) method [2, 4, 6, 19]. However, compared with explicit methods, implicit methods require more additional computational efforts and cost much more time. Therefore, many scholars pay attention to find an explicit method which will converge in the strong sense when the coefficients of SDEs satisfy the super-linear growth condition. At present, there are some effective methods, such as the stopped Euler method [12], the tamed Euler method [1, 8, 26], the truncated Euler-Maruyama method [5, 16, 18], the partially truncated Euler-Maruyama method [3] and the modified truncated Euler-Maruyama method [10]. In this paper, we develop the truncated Euler-Maruyama (EM) method for SDEPCAs, and consider the strong convergence theory under the local Lipschitz condition plus the Khasminskii-type condition. The order of convergence is obtained.

Guo et al. [3] investigated the mean square exponential stability of the partially truncated Euler-Maruyama method for SDEs. Later, Lan and Xia [10] gave the exponential stability of the modified truncated Euler-Maruyama method for SDEs. Hu et al. [5] obtained the asymptotic stability of the truncated Euler-Maruyama method for SDEs. In this paper, we show that the truncated Euler-Maruyama method preserves the exponential mean square stability of the SDEPCAs.

The rest of this article is organized as follows. Section 2 introduces some basic assumptions, definitions and properties of the exact solution. We construct the truncated Euler-Maruyama method in Section 3. The $p$-th moment boundedness of the numerical solutions and the convergence theorem are presented in Section 4. Section 5 obtains the convergence order and Section 6 shows the exponential stabilities of both the SDEPCAs and the numerical solutions. Numerical simulations are provided to verify the analytical theory in Section 7. 


\section{Analysis of exact solutions}

Throughout this paper, unless otherwise specified, we will use the following notations. If $\mathbf{A}$ is a vector or matrix, its transpose is denoted by $\mathbf{A}^{\mathrm{T}}$. If $x \in \mathbf{R}^{n}$, then $|x|$ is the Euclidean norm. If $\mathbf{A}$ is a matrix, we let $|\mathbf{A}|=\sqrt{\operatorname{trace}\left(\mathbf{A}^{\mathrm{T}} \mathbf{A}\right)}$ be its trace norm. For two real numbers $a$ and $b$, we use $a \vee b$ and $a \wedge b$ to denote $\max (a, b)$ and $\min (a, b)$, respectively. If $D$ is a set, its indicator function is denoted by $I_{D}$. Moreover, let $(\Omega, \mathcal{F}, \mathbb{P})$ be a complete probability space with a filtration $\left\{\mathcal{F}_{t}\right\}_{t \geq 0}$ satisfying the usual conditions (that is, it is right continuous and increasing while $\mathcal{F}_{0}$ contains all $\mathbb{P}$-null sets), and let $\mathbb{E}$ denote the expectation corresponding to $\mathbb{P}$. Denote $\mathcal{L}^{p}\left([0, T] ; \mathbf{R}^{n \times d}\right)$ by the family of $\mathbf{R}^{n \times d}$-valued $\mathcal{F}_{t}$-adapted processes $\{f(t)\}_{0 \leq t \leq T}$ such that $\int_{0}^{T}|f(t)|^{p} d t<\infty$, a.s.. Denote $\mathcal{L}^{p}\left([0, \infty), \mathbf{R}^{n \times d}\right)$ by the family of the processes $\{f(t)\}_{t \geq 0}$ such that for every $T>0,\{f(t)\}_{0 \leq t \leq T} \in \mathcal{L}^{p}\left([0, T] ; \mathbf{R}^{n \times d}\right)$. Let $B(t)$ be a $d$-dimensional Brownian motion defined on the space. Consider the $n$-dimensional SDEPCAs

$$
d x(t)=f(x(t), x([t])) d t+g(x(t), x([t])) d B(t)
$$

on $t \geq 0$ with the initial value $x(0)=x_{0} \in \mathbf{R}^{n}$, where $f: \mathbf{R}^{n} \times \mathbf{R}^{n} \rightarrow \mathbf{R}^{n}, g: \mathbf{R}^{n} \times \mathbf{R}^{n} \rightarrow$ $\mathbf{R}^{n \times d}$ are measurable functions and $[t]$ denotes the greatest-integer part of $t$. Eq. (2.1) is equivalent to the following stochastic integral equation:

$$
x(t)=x_{0}+\int_{0}^{t} f(x(s), x([s])) d s+\int_{0}^{t} g(x(s), x([s])) d B(s), \quad \forall t \geq 0 .
$$

Moreover, we also require that the coefficients $f$ and $g$ are sufficiently smooth. To guarantee the existence of the global solution, we present the following assumptions.

Assumption 2.1. (The local Lipschitz condition.) For every integer $R \geq 0$, there exists a positive constant $L_{R}$, such that

$$
|f(x, y)-f(\bar{x}, \bar{y})| \vee|g(x, y)-g(\bar{x}, \bar{y})| \leq L_{R}(|x-\bar{x}|+|y-\bar{y}|)
$$

for all $x, \bar{x}, y, \bar{y} \in \mathbf{R}^{n},|x| \vee|y| \vee|\bar{x}| \vee|\bar{y}| \leq R$.

Assumption 2.2. (Khasminskii-type condition.) There are two constants $K>0$ and $p \geq 2$, such that

$$
x^{\mathrm{T}} f(x, y)+\frac{p-1}{2}|g(x, y)|^{2} \leq K\left(1+|x|^{2}+|y|^{2}\right)
$$

for all $x, y \in \mathbf{R}^{n}$.

The definition of the exact solution of (2.1) is given in the following.

Definition 2.1. ([24]) An $\mathbf{R}^{n}$-valued stochastic process $\{x(t)\}$ is called a solution of Eq. (2.1) on $[0, \infty)$ if it has the following properties: 
(1) $\{x(t)\}$ is continuous on $[0, \infty)$ and $\mathcal{F}_{t}$-adapted;

(2) $\{f(x(t), x([t]))\} \in \mathcal{L}^{1}\left([0, \infty), \mathbf{R}^{n}\right)$ and $\{g(x(t), x([t]))\} \in \mathcal{L}^{2}\left([0, \infty), \mathbf{R}^{n \times d}\right)$;

(3) Eq. (2.2) is satisfied on each interval $[n, n+1) \subset[0, \infty)$ with integer end-points almost surely.

A solution $\{x(t)\}$ is said to be unique if any other solution $\{\bar{x}(t)\}$ is indistinguishable from $\{x(t)\}$, that is

$$
\mathbb{P}\{x(t)=\bar{x}(t), t \in[0, \infty)\}=1 .
$$

We state a known result in [24] as a lemma for the use of this paper.

Lemma 2.1. Under Assumptions 2.1 and 2.2, the SDEPCAs (2.1) have a unique global solution $x(t)$. Moreover,

$$
\sup _{0 \leq t \leq T} \mathbb{E}|x(t)|^{p}<\infty, \quad \forall T>0 .
$$

Lemma 2.2. Let Assumptions 2.1 and 2.2 hold. Define the stopping time

$$
\tau_{R}=\inf \{t \geq 0:|x(t)| \geq R\},
$$

where throughout this paper we set $\inf \emptyset=\infty$. Then for any $R>\left|x_{0}\right|$, we have

$$
\mathbb{P}\left\{\tau_{R} \leq T\right\} \leq \frac{C}{R^{2}},
$$

where $C$ stands for a generic positive real constant dependent on $T, K, x_{0}$, but independent of $R$ and its value may change between occurrences.

Proof. By the Itô formula and Assumption 2.2, we obtain

$$
\begin{aligned}
\mathbb{E}\left|x\left(t \wedge \tau_{R}\right)\right|^{2} & \leq\left|x_{0}\right|^{2}+\mathbb{E} \int_{0}^{t \wedge \tau_{R}} 2 K\left(1+|x(s)|^{2}+|x([s])|^{2}\right) d s \\
& \leq\left|x_{0}\right|^{2}+2 K T+4 K \int_{0}^{t} \sup _{0 \leq u \leq s} \mathbb{E}\left|x\left(u \wedge \tau_{R}\right)\right|^{2} d s, \quad \forall 0 \leq t \leq T .
\end{aligned}
$$

For any $0 \leq t \leq T$, we have

$$
\sup _{0 \leq t \leq T} \mathbb{E}\left|x\left(t \wedge \tau_{R}\right)\right|^{2} \leq\left|x_{0}\right|^{2}+2 K T+4 K \int_{0}^{T} \sup _{0 \leq u \leq s} \mathbb{E}\left|x\left(u \wedge \tau_{R}\right)\right|^{2} d s .
$$

Applying the Gronwall inequality, we get

$$
\mathbb{E}\left|x\left(T \wedge \tau_{R}\right)\right|^{2} \leq C .
$$

This implies

$$
R^{2} \mathbb{P}\left\{\tau_{R} \leq T\right\} \leq C
$$

and the assertion follows. 


\section{The truncated Euler-Maruyama method}

To define the truncated Euler-Maruyama numerical solutions, we choose a strictly increasing continuous function $\mu:[1, \infty) \rightarrow \mathbf{R}_{+}$such that $\mu(r) \rightarrow \infty$ as $r \rightarrow \infty$ and

$$
\sup _{|x| \vee|y| \leq r}(|f(x, y)| \vee|g(x, y)|) \leq \mu(r), \quad \forall r \geq 1 .
$$

The inverse function of $\mu$ is denoted by $\mu^{-1}$ and we see that $\mu^{-1}$ is a strictly increasing continuous function. We also choose a constant $\hat{h} \geq 1$ and a strictly decreasing function $h:(0,1] \rightarrow[\mu(1), \infty)$ such that

$$
\lim _{\Delta \rightarrow 0} h(\Delta)=\infty, \quad \Delta^{\frac{1}{4}} h(\Delta) \leq \hat{h}, \quad \Delta \in(0,1] .
$$

It follows from $h(\Delta) \geq \mu(1)$ that $\mu^{-1}(h(\Delta)) \geq 1$.

For a given step size $\Delta \in(0,1]$, define the mapping $\pi_{\Delta}: \mathbf{R}^{n} \rightarrow \mathbf{R}^{n}$ by

$$
\pi_{\Delta}(x)=\left(|x| \wedge \mu^{-1}(h(\Delta))\right) \frac{x}{|x|},
$$

where we set $\frac{x}{|x|}=0$ when $x=0$. We then define the truncated functions

$$
f_{\Delta}(x, y)=f\left(\pi_{\Delta}(x), \pi_{\Delta}(y)\right), \quad g_{\Delta}(x, y)=g\left(\pi_{\Delta}(x), \pi_{\Delta}(y)\right), \quad \forall x, y \in \mathbf{R}^{n} .
$$

It is easy to see that

$$
\left|f_{\Delta}(x, y)\right| \vee\left|g_{\Delta}(x, y)\right| \leq \mu\left(\mu^{-1}(h(\Delta))\right)=h(\Delta), \quad \forall x, y \in \mathbf{R}^{n} .
$$

The following lemma shows that these truncated functions preserve the Khasminskii-type condition (2.4) for all $\Delta \in(0,1]$.

Lemma 3.1. Let Assumption 2.2 hold. Then for all $\Delta \in(0,1]$, we have

$$
x^{\mathrm{T}} f_{\Delta}(x, y)+\frac{p-1}{2}\left|g_{\Delta}(x, y)\right|^{2} \leq 3 K\left(1+|x|^{2}+|y|^{2}\right), \quad x, y \in \boldsymbol{R}^{n} .
$$

Proof. Case 1. If $x \in \mathbf{R}^{n}$ with $|x| \leq \mu^{-1}(h(\Delta))$, then $\pi_{\Delta}(x)=x$. Moreover, for any $y \in \mathbf{R}^{n}$, we have

Therefore

$$
\left|\pi_{\Delta}(y)\right|=\left|\left(|y| \wedge \mu^{-1}(h(\Delta))\right) \frac{y}{|y|}\right| \leq|y| .
$$

$$
\begin{aligned}
& x^{\mathrm{T}} f_{\Delta}(x, y)+\frac{p-1}{2}\left|g_{\Delta}(x, y)\right|^{2} \\
= & \pi_{\Delta}^{\mathrm{T}}(x) f\left(\pi_{\Delta}(x), \pi_{\Delta}(y)\right)+\frac{p-1}{2}\left|g\left(\pi_{\Delta}(x), \pi_{\Delta}(y)\right)\right|^{2} \\
\leq & K\left(1+\left|\pi_{\Delta}(x)\right|^{2}+\left|\pi_{\Delta}(y)\right|^{2}\right) \\
\leq & K\left(1+|x|^{2}+|y|^{2}\right) .
\end{aligned}
$$


Case 2. If $x \in \mathbf{R}^{n}$ with $|x|>\mu^{-1}(h(\Delta)) \geq 1$, then

$$
\pi_{\Delta}(x)=\left(|x| \wedge \mu^{-1}(h(\Delta))\right) \frac{x}{|x|}=\mu^{-1}(h(\Delta)) \frac{x}{|x|} .
$$

For any $y \in \mathbf{R}^{n}$, we have

$$
\begin{aligned}
& x^{\mathrm{T}} f_{\Delta}(x, y)+\frac{p-1}{2}\left|g_{\Delta}(x, y)\right|^{2} \\
= & \pi_{\Delta}^{\mathrm{T}}(x) f\left(\pi_{\Delta}(x), \pi_{\Delta}(y)\right)+\frac{p-1}{2}\left|g\left(\pi_{\Delta}(x), \pi_{\Delta}(y)\right)\right|^{2} \\
& +\left(x-\pi_{\Delta}(x)\right)^{\mathrm{T}} f\left(\pi_{\Delta}(x), \pi_{\Delta}(y)\right) \\
\leq & K\left(1+\left|\pi_{\Delta}(x)\right|^{2}+\left|\pi_{\Delta}(y)\right|^{2}\right) \\
& +\left(\frac{|x|}{\mu^{-1}(h(\Delta))}-1\right) \pi_{\Delta}^{\mathrm{T}}(x) f\left(\pi_{\Delta}(x), \pi_{\Delta}(y)\right) .
\end{aligned}
$$

By Assumption 2.2, we obtain

$$
\pi_{\Delta}^{\mathrm{T}}(x) f\left(\pi_{\Delta}(x), \pi_{\Delta}(y)\right) \leq K\left(1+\left|\pi_{\Delta}(x)\right|^{2}+\left|\pi_{\Delta}(y)\right|^{2}\right) .
$$

Thus

$$
\begin{aligned}
& x^{\mathrm{T}} f_{\Delta}(x, y)+\frac{p-1}{2}\left|g_{\Delta}(x, y)\right|^{2} \\
\leq & \frac{|x|}{\mu^{-1}(h(\Delta))} K\left(1+\left|\pi_{\Delta}(x)\right|^{2}+\left|\pi_{\Delta}(y)\right|^{2}\right) \\
\leq & K\left(|x|+|x|^{2}+|x||y|\right) \\
\leq & 3 K\left(1+|x|^{2}+|y|^{2}\right) .
\end{aligned}
$$

The proof is completed.

Let $\Delta=\frac{1}{m}$ be a given step size with integer $m>0$. Let $T=N \Delta, N \in \mathbb{N}^{+}$and the grid points $t_{k}$ can be defined by $t_{k}=k \Delta, k=0, \ldots, N-1$. Since for each $k$, there exist $s \in \mathbb{N}$ and $l \in\{0, \ldots, m-1\}$ such that $k=s m+l$. The truncated Euler-Maruyama method is given by

$$
\begin{aligned}
X_{\Delta}\left(t_{s m+l+1}\right)= & X_{\Delta}\left(t_{s m+l}\right)+f_{\Delta}\left(X_{\Delta}\left(t_{s m+l}\right), X_{\Delta}\left(t_{s m}\right)\right) \Delta \\
& +g_{\Delta}\left(X_{\Delta}\left(t_{s m+l}\right), X_{\Delta}\left(t_{s m}\right)\right) \Delta B_{s m+l},
\end{aligned}
$$

where $X_{\Delta}(0)=x_{0}, \Delta B_{s m+l}=B\left(t_{s m+l+1}\right)-B\left(t_{s m+l}\right)$ and $X_{\Delta}\left(t_{s m+l}\right)$ is the approximation to $x(t)$ at $t=t_{s m+l}$.

The two types of continuous-time truncated Euler-Maruyama solutions are defined as follows. For any $t \geq 0$, 


$$
\begin{aligned}
& \bar{x}_{\Delta}(t)=\sum_{s m+l=0}^{\infty} X_{\Delta}\left(t_{s m+l}\right) I_{\left[t_{s m+l}, t_{s m+l+1}\right)}(t) \\
& x_{\Delta}(t)=x_{0}+\int_{0}^{t} f_{\Delta}\left(\bar{x}_{\Delta}(s), \bar{x}_{\Delta}([s])\right) d s+\int_{0}^{t} g_{\Delta}\left(\bar{x}_{\Delta}(s), \bar{x}_{\Delta}([s])\right) d B(s) .
\end{aligned}
$$

\section{Convergence of the truncated Euler-Maruyama method}

In this section, we will show that the truncated Euler-Maruyama method converges to the exact solution of Eq. (2.1). In order to get our conclusions, we need some useful lemmas. The first lemma shows that $x_{\Delta}(t)$ and $\bar{x}_{\Delta}(t)$ are arbitrarily closed to each other.

Lemma 4.1. Assume that Assumptions 2.1,2.2 and (3.2) hold. For any $\Delta \in(0,1]$, we have

$$
\mathbb{E}\left|x_{\Delta}(t)-\bar{x}_{\Delta}(t)\right|^{p} \leq C \Delta^{\frac{p}{2}}(h(\Delta))^{p}, \quad \forall t \geq 0,
$$

where $C$ is a positive constant that depends only on $p$, but is independent of $\Delta$ and its value may change between occurrences. Consequently,

$$
\lim _{\Delta \rightarrow 0} \mathbb{E}\left|x_{\Delta}(t)-\bar{x}_{\Delta}(t)\right|^{p}=0, \quad \forall t \geq 0 .
$$

Proof. For any fixed $\Delta \in(0,1]$ and $t \in\left[t_{s m+l}, t_{s m+l+1}\right)$, we have $\bar{x}_{\Delta}(t)=X_{\Delta}\left(t_{s m+l}\right)$ and

$$
\begin{aligned}
& x_{\Delta}(t)-\bar{x}_{\Delta}(t) \\
= & \int_{t_{s m+l}}^{t} f_{\Delta}\left(\bar{x}_{\Delta}(s), \bar{x}_{\Delta}([s])\right) d s+\int_{t_{s m+l}}^{t} g_{\Delta}\left(\bar{x}_{\Delta}(s), \bar{x}_{\Delta}([s])\right) d B(s) .
\end{aligned}
$$

By the properties of the Itô integral, Hölder inequality, Theorem 1.7.1 in [15] and (3.4), we then obtain

$$
\begin{aligned}
& \mathbb{E}\left|x_{\Delta}(t)-\bar{x}_{\Delta}(t)\right|^{p} \\
\leq & 2^{p-1} \Delta^{p-1} \mathbb{E} \int_{t_{s m+l}}^{t}\left|f_{\Delta}\left(\bar{x}_{\Delta}(s), \bar{x}_{\Delta}([s])\right)\right|^{p} d s \\
& +2^{p-1}\left(\frac{p(p-1)}{2}\right)^{\frac{p}{2}} \Delta^{\frac{p-2}{2}} \mathbb{E} \int_{t_{s m+l}}^{t}\left|g_{\Delta}\left(\bar{x}_{\Delta}(s), \bar{x}_{\Delta}([s])\right)\right|^{p} d s \\
\leq & C \Delta^{\frac{p}{2}}(h(\Delta))^{p},
\end{aligned}
$$

where

$$
C=2^{p-1}\left(1+\left(\frac{p(p-1)}{2}\right)^{\frac{p}{2}}\right) .
$$


Moreover, by (3.2), we have

$$
\mathbb{E}\left|x_{\Delta}(t)-\bar{x}_{\Delta}(t)\right|^{p} \leq C \Delta^{\frac{p}{2}}(h(\Delta))^{p} \leq C \hat{h}^{p} \Delta^{\frac{p}{4}} .
$$

We obtain (4.2) from (4.5) as $\Delta \rightarrow 0$.

Before giving the $p$-th moment boundedness of $x_{\Delta}(t)$, we define the stopping time

$$
\rho_{R}=\inf \left\{t \geq 0:\left|x_{\Delta}(t)\right| \geq R\right\}
$$

and present the following lemma.

Lemma 4.2. Assume that Assumptions 2.1,2.2 and (3.2) hold. For any $R>\left|x_{0}\right|$ and $\Delta \in(0,1]$, we have

$$
\mathbb{P}\left\{\rho_{R} \leq T\right\} \leq \frac{C}{R^{2}},
$$

where $C$ is a constant independent of $R$ and $\Delta$.

Proof. By the Itô formula, we have

$$
\begin{aligned}
& \mathbb{E}\left|x_{\Delta}\left(t \wedge \rho_{R}\right)\right|^{2} \\
= & \left|x_{0}\right|^{2}+2 \mathbb{E} \int_{0}^{t \wedge \rho_{R}}\left(x_{\Delta}^{\mathrm{T}}(s) f_{\Delta}\left(\bar{x}_{\Delta}(s), \bar{x}_{\Delta}([s])\right)+\frac{1}{2}\left|g_{\Delta}\left(\bar{x}_{\Delta}(s), \bar{x}_{\Delta}([s])\right)\right|^{2}\right) d s \\
= & \left|x_{0}\right|^{2}+2 \mathbb{E} \int_{0}^{t \wedge \rho_{R}}\left(\bar{x}_{\Delta}^{\mathrm{T}}(s) f_{\Delta}\left(\bar{x}_{\Delta}(s), \bar{x}_{\Delta}([s])\right)+\frac{1}{2}\left|g_{\Delta}\left(\bar{x}_{\Delta}(s), \bar{x}_{\Delta}([s])\right)\right|^{2}\right) d s \\
& +2 \mathbb{E} \int_{0}^{t \wedge \rho_{R}}\left(\left(x_{\Delta}(s)-\bar{x}_{\Delta}(s)\right)^{\mathrm{T}} f_{\Delta}\left(\bar{x}_{\Delta}(s), \bar{x}_{\Delta}([s])\right)\right) d s .
\end{aligned}
$$

Using Lemma 3.1, we have

$$
\begin{aligned}
& 2 \mathbb{E} \int_{0}^{t \wedge \rho_{R}}\left(\bar{x}_{\Delta}^{\mathrm{T}}(s) f_{\Delta}\left(\bar{x}_{\Delta}(s), \bar{x}_{\Delta}([s])\right)+\frac{1}{2}\left|g_{\Delta}\left(\bar{x}_{\Delta}(s), \bar{x}_{\Delta}([s])\right)\right|^{2}\right) d s \\
\leq & 6 K \mathbb{E} \int_{0}^{t \wedge \rho_{R}}\left(1+\left|\bar{x}_{\Delta}(s)\right|^{2}+\left|\bar{x}_{\Delta}([s])\right|^{2}\right) d s \\
\leq & 6 K T+12 K \int_{0}^{t} \sup _{0 \leq u \leq s} \mathbb{E}\left|\bar{x}_{\Delta}\left(u \wedge \rho_{R}\right)\right|^{2} d s .
\end{aligned}
$$

By (3.4) and Lemma 4.1, the third term of (4.8) yields

$$
2 \mathbb{E} \int_{0}^{t \wedge \rho_{R}}\left(\left(x_{\Delta}(s)-\bar{x}_{\Delta}(s)\right)^{\mathrm{T}} f_{\Delta}\left(\bar{x}_{\Delta}(s), \bar{x}_{\Delta}([s])\right)\right) d s \leq 2 T C \hat{h}^{2} .
$$

Combining (4.9) and (4.10), we have

$$
\begin{aligned}
& \mathbb{E}\left|x_{\Delta}\left(t \wedge \rho_{R}\right)\right|^{2} \\
\leq & \left|x_{0}\right|^{2}+6 K T+2 T C \hat{h}^{2}+12 K \int_{0}^{t} \sup _{0 \leq u \leq s} \mathbb{E}\left|\bar{x}_{\Delta}\left(u \wedge \rho_{R}\right)\right|^{2} d s .
\end{aligned}
$$


For any $0 \leq t \leq T$, applying the Gronwall inequality, we obtain

$$
\sup _{0 \leq t \leq T} \mathbb{E}\left|x_{\Delta}\left(t \wedge \rho_{R}\right)\right|^{2} \leq C
$$

Then $\mathbb{E}\left|x_{\Delta}\left(T \wedge \rho_{R}\right)\right|^{2} \leq C$, which implies that $R^{2} \mathbb{P}\left\{\rho_{R} \leq T\right\} \leq C$. We complete the proof.

Lemma 4.3. Assume that Assumptions 2.1 and 2.2 hold, then there exists a positive constant $C$ related to $p, T, x_{0}$ but independent of $\Delta$ such that

$$
\sup _{0<\Delta \leq 1} \sup _{0 \leq t \leq T} \mathbb{E}\left|x_{\Delta}(t)\right|^{p} \leq C .
$$

Proof. For any fixed $\Delta \in(0,1]$, by the Itô formula, Hölder inequality, Lemma 4.1, (3.4) and (3.2), we derive

$$
\begin{aligned}
\mathbb{E}\left|x_{\Delta}(t)\right|^{p} \leq & \left|x_{0}\right|^{p}+p \mathbb{E} \int_{0}^{t}\left|x_{\Delta}(s)\right|^{p-2}\left[x_{\Delta}^{\mathrm{T}}(s) f_{\Delta}\left(\bar{x}_{\Delta}(s), \bar{x}_{\Delta}([s])\right)\right. \\
& \left.+\frac{p-1}{2}\left|g_{\Delta}\left(\bar{x}_{\Delta}(s), \bar{x}_{\Delta}([s])\right)\right|^{2}\right] d s \\
\leq & \left|x_{0}\right|^{p}+2 p K \mathbb{E} \int_{0}^{t}\left|x_{\Delta}(s)\right|^{p-2}\left(1+\left|\bar{x}_{\Delta}(s)\right|^{2}+\left|\bar{x}_{\Delta}([s])\right|^{2}\right) d s \\
& +p \mathbb{E} \int_{0}^{t}\left|x_{\Delta}(s)\right|^{p-2}\left|x_{\Delta}(s)-\bar{x}_{\Delta}(s)\right|\left|f_{\Delta}\left(\bar{x}_{\Delta}(s), \bar{x}_{\Delta}([s])\right)\right| d s \\
\leq & \left|x_{0}\right|^{p}+T\left(C \hat{h}+2^{\frac{p}{2}+1} K\right) \\
& +\left((p-2)(1+2 K)+2^{\frac{p}{2}+2} K\right) \int_{0}^{t} \sup _{0 \leq u \leq s} \mathbb{E}\left|x_{\Delta}(u)\right|^{p} d s .
\end{aligned}
$$

Applying the Gronwall inequality, we obtain

$$
\sup _{0 \leq t \leq T} \mathbb{E}\left|x_{\Delta}(t)\right|^{p} \leq C
$$

Since (4.15) holds for any $\Delta \in(0,1]$ and $C$ is independent of $\Delta$, we obtain the required assertion (4.13).

Theorem 4.1. Let Assumptions 2.1 and 2.2 hold. Then for any $q \in[2, p)$, we obtain

$$
\lim _{\Delta \rightarrow 0} \mathbb{E}\left|x_{\Delta}(T)-x(T)\right|^{q}=0 .
$$

Proof. Let $\tau_{R}$ and $\rho_{R}$ be the same as before. Set

$$
\theta_{R}=\tau_{R} \wedge \rho_{R}, \quad e_{\Delta}(T)=x(T)-x_{\Delta}(T) .
$$


Using the Young inequality, we derive that for any $\delta>0$,

$$
\begin{aligned}
\mathbb{E}\left|e_{\Delta}(T)\right|^{q} & =\mathbb{E}\left(\left|e_{\Delta}(T)\right|{ }^{q} I_{\left\{\theta_{R}>T\right\}}\right)+\mathbb{E}\left(\left|e_{\Delta}(T)\right|^{q} I_{\left\{\theta_{R} \leq T\right\}}\right) \\
& \leq \mathbb{E}\left(\left|e_{\Delta}(T)\right|^{q} I_{\left\{\theta_{R}>T\right\}}\right)+\frac{q \delta}{p} \mathbb{E}\left|e_{\Delta}(T)\right|^{p}+\frac{p-q}{p \delta^{\frac{q}{(p-q)}}} \mathbb{P}\left\{\theta_{R} \leq T\right\} .
\end{aligned}
$$

By Lemmas 2.1 and 4.3, we obtain

$$
\mathbb{E}\left|e_{\Delta}(T)\right|^{p} \leq C .
$$

Applying Lemmas 2.2 and 4.2, we have

$$
\mathbb{P}\left\{\theta_{R} \leq T\right\} \leq \mathbb{P}\left\{\tau_{R} \leq T\right\}+\mathbb{P}\left\{\rho_{R} \leq T\right\} \leq \frac{C}{R^{2}} .
$$

By basic inequalities, we have

$$
\begin{aligned}
& \mathbb{E}\left|e_{\Delta}\left(t \wedge \theta_{R}\right)\right|^{q} \\
= & \mathbb{E} \mid \int_{0}^{t \wedge \theta_{R}}\left(f(x(s), x([s]))-f_{\Delta}\left(\bar{x}_{\Delta}(s), \bar{x}_{\Delta}([s])\right)\right) d s \\
& \quad+\left.\int_{0}^{t \wedge \theta_{R}}\left(g(x(s), x([s]))-g_{\Delta}\left(\bar{x}_{\Delta}(s), \bar{x}_{\Delta}([s])\right)\right) d B(s)\right|^{q} \\
\leq & J_{1}+J_{2},
\end{aligned}
$$

where

$$
\begin{gathered}
J_{1}=(2 T)^{q-1} \mathbb{E} \int_{0}^{t} \mid f\left(x\left(s \wedge \theta_{R}\right), x\left(\left[s \wedge \theta_{R}\right]\right)\right) \\
-\left.f_{\Delta}\left(\bar{x}_{\Delta}\left(s \wedge \theta_{R}\right), \bar{x}_{\Delta}\left(\left[s \wedge \theta_{R}\right]\right)\right)\right|^{q} d s, \\
J_{2}=2^{q-1} T^{\frac{q}{2}-1}\left(\frac{q(q-1)}{2}\right)^{\frac{q}{2}} \mathbb{E} \int_{0}^{t}\left[\mid g\left(x\left(s \wedge \theta_{R}\right), x\left(\left[s \wedge \theta_{R}\right]\right)\right)\right. \\
\left.-\left.g_{\Delta}\left(\bar{x}_{\Delta}\left(s \wedge \theta_{R}\right), \bar{x}_{\Delta}\left(\left[s \wedge \theta_{R}\right]\right)\right)\right|^{q}\right] d s .
\end{gathered}
$$

Applying basic inequalities, we have

$$
J_{1} \leq(6 T)^{q-1}\left(J_{11}+J_{12}+J_{13}\right)
$$

where

$$
\begin{aligned}
& J_{11}=\mathbb{E} \int_{0}^{t}\left|f\left(x\left(s \wedge \theta_{R}\right), x\left(\left[s \wedge \theta_{R}\right]\right)\right)-f\left(x_{\Delta}\left(s \wedge \theta_{R}\right), x_{\Delta}\left(\left[s \wedge \theta_{R}\right]\right)\right)\right|^{q} d s, \\
& J_{12}=\mathbb{E} \int_{0}^{t}\left|f\left(x_{\Delta}\left(s \wedge \theta_{R}\right), x_{\Delta}\left(\left[s \wedge \theta_{R}\right]\right)\right)-f\left(\bar{x}_{\Delta}\left(s \wedge \theta_{R}\right), \bar{x}_{\Delta}\left(\left[s \wedge \theta_{R}\right]\right)\right)\right|^{q} d s, \\
& J_{13}=\mathbb{E} \int_{0}^{t}\left|f\left(\bar{x}_{\Delta}\left(s \wedge \theta_{R}\right), \bar{x}_{\Delta}\left(\left[s \wedge \theta_{R}\right]\right)\right)-f_{\Delta}\left(\bar{x}_{\Delta}\left(s \wedge \theta_{R}\right), \bar{x}_{\Delta}\left(\left[s \wedge \theta_{R}\right]\right)\right)\right|^{q} d s .
\end{aligned}
$$


By the local Lipschitz condition and basic inequalities, we have

$$
\begin{aligned}
J_{11} & \leq 2^{q-1} L_{R}^{q} \int_{0}^{t}\left[\mathbb{E}\left|x\left(s \wedge \theta_{R}\right)-x_{\Delta}\left(s \wedge \theta_{R}\right)\right|^{q}+\mathbb{E}\left|x\left(\left[s \wedge \theta_{R}\right]\right)-x_{\Delta}\left(\left[s \wedge \theta_{R}\right]\right)\right|^{q}\right] d s \\
& \leq\left(2 L_{R}\right)^{q} \int_{0}^{t} \sup _{0 \leq u \leq s} \mathbb{E}\left|x\left(u \wedge \theta_{R}\right)-x_{\Delta}\left(u \wedge \theta_{R}\right)\right|^{q} d s .
\end{aligned}
$$

Similarly to $J_{11}$, applying Lemma 4.1, we derive

$$
\begin{aligned}
J_{12} & \leq 2^{q-1} L_{R}^{q} \int_{0}^{t} \mathbb{E}\left[\left|x_{\Delta}\left(s \wedge \theta_{R}\right)-\bar{x}_{\Delta}\left(s \wedge \theta_{R}\right)\right|^{q}+\mathbb{E}\left|x_{\Delta}\left(\left[s \wedge \theta_{R}\right]\right)-\bar{x}_{\Delta}\left(\left[s \wedge \theta_{R}\right]\right)\right|^{q}\right] d s \\
& \leq\left(2 L_{R}\right)^{q} \int_{0}^{t} \sup _{0 \leq u \leq s} \mathbb{E}\left|x_{\Delta}\left(u \wedge \theta_{R}\right)-\bar{x}_{\Delta}\left(u \wedge \theta_{R}\right)\right|^{q} d s \\
& \leq\left(2 L_{R}\right)^{q} C T \Delta^{\frac{q}{2}}(h(\Delta))^{q} \leq C \hat{h}^{q} L_{R}^{q} \Delta^{\frac{q}{4}} .
\end{aligned}
$$

By local Lipschitz condition, Hölder inequality, Chebyshev's inequality and Lemma 4.3, we have

$$
\begin{aligned}
J_{13} & \leq\left(2 L_{R}\right)^{q} \int_{0}^{t} \sup _{0 \leq u \leq s} \mathbb{E}\left|\bar{x}_{\Delta}\left(u \wedge \theta_{R}\right)-\pi_{\Delta}\left(\bar{x}_{\Delta}\left(u \wedge \theta_{R}\right)\right)\right|^{q} d s \\
& \leq\left(2 L_{R}\right)^{q} \int_{0}^{t} \sup _{0 \leq u \leq s} \mathbb{E}\left(\left|\bar{x}_{\Delta}\left(u \wedge \theta_{R}\right)\right|^{q} I_{\left\{\bar{x}_{\Delta}\left(u \wedge \theta_{R}\right)>\mu^{-1}(h(\Delta))\right\}}\right) d s \\
& \leq\left(2 L_{R}\right)^{q} \int_{0}^{t} \sup _{0 \leq u \leq s}\left(\mathbb{E}\left|\bar{x}_{\Delta}\left(u \wedge \theta_{R}\right)\right|^{p}\right)^{\frac{q}{p}}\left(\mathbb{P}\left\{\bar{x}_{\Delta}\left(u \wedge \theta_{R}\right)>\mu^{-1}(h(\Delta))\right\}\right)^{\frac{p-q}{p}} d s \\
& \leq C\left(2 L_{R}\right)^{q} \int_{0}^{t} \sup _{0 \leq u \leq s}\left(\frac{\mathbb{E}\left|\bar{x}_{\Delta}\left(u \wedge \theta_{R}\right)\right|^{p}}{\left|\mu^{-1}(h(\Delta))\right|^{p-q}}\right) d s \\
& \leq \frac{C\left(2 L_{R}\right)^{q}}{\left|\mu^{-1}(h(\Delta))\right|^{p-q}}
\end{aligned}
$$

Combining the Eqs. (4.22) and (4.24)-(4.26), we obtain

$$
\begin{gathered}
J_{1} \leq(6 T)^{q-1}\left[\left(2 L_{R}\right)^{q} \int_{0}^{t} \sup _{0 \leq u \leq s} \mathbb{E}\left|x\left(u \wedge \theta_{R}\right)-x_{\Delta}\left(u \wedge \theta_{R}\right)\right|^{q} d s\right. \\
\left.+C \hat{h} L_{R}^{q} \Delta^{\frac{q}{4}}+\frac{C\left(2 L_{R}\right)^{q}}{\left|\mu^{-1}(h(\Delta))\right|^{p-q}}\right] .
\end{gathered}
$$

Similarly to $J_{1}$, we have

$$
\begin{aligned}
& J_{2} \leq 6^{q-1} T^{\frac{q}{2}-1}\left(\frac{q(q-1)}{2}\right)^{\frac{q}{2}}\left[\left(2 L_{R}\right)^{q} \int_{0}^{t} \sup _{0 \leq u \leq s} \mathbb{E}\left|x\left(u \wedge \theta_{R}\right)-x_{\Delta}\left(u \wedge \theta_{R}\right)\right|^{q} d s\right. \\
& \left.+C \hat{h}^{q} L_{R}^{q} \Delta^{\frac{q}{4}}+\frac{C\left(2 L_{R}\right)^{q}}{\left|\mu^{-1}(h(\Delta))\right|^{p-q}}\right]
\end{aligned}
$$


Hence,

$$
\begin{aligned}
& \mathbb{E}\left|e_{\Delta}\left(t \wedge \theta_{R}\right)\right|^{q} \\
\leq & \left((6 T)^{q-1}+6^{q-1} T^{\frac{q}{2}-1}\left(\frac{q(q-1)}{2}\right)^{\frac{q}{2}}\right) \\
& \times\left(\left(2 L_{R}\right)^{q} \int_{0}^{t} \sup _{0 \leq u \leq s} \mathbb{E}\left|e\left(u \wedge \theta_{R}\right)\right|^{q} d s+C \hat{h}^{q} L_{R}^{q} \Delta^{\frac{q}{4}}+\frac{C\left(2 L_{R}\right)^{q}}{\left|\mu^{-1}(h(\Delta))\right|^{p-q}}\right) .
\end{aligned}
$$

By the Gronwall inequality, we have

$$
\mathbb{E}\left|e_{\Delta}\left(t \wedge \theta_{R}\right)\right|^{q} \leq C L_{R}^{q}\left(\Delta^{\frac{q}{4}}+\frac{1}{\left|\mu^{-1}(h(\Delta))\right|^{p-q}}\right) e^{C L_{R}^{q}} .
$$

Submitting Eq. (4.30) into Eq. (4.17), we obtain

$$
\mathbb{E}\left|e_{\Delta}(T)\right|^{q} \leq C L_{R}^{q}\left(\Delta^{\frac{q}{4}}+\frac{1}{\left|\mu^{-1}(h(\Delta))\right|^{p-q}}\right) e^{C L_{R}^{q}}+\frac{C q \delta}{p}+\frac{C(p-q)}{p R^{2} \delta^{\frac{q}{(p-q)}}} .
$$

For any $\varepsilon>0$, we can choose $\delta>0$ such that $\frac{C q \delta}{p}<\frac{\varepsilon}{3}$. Then we choose $R$ sufficiently large for

$$
\frac{C(p-q)}{p R^{2} \delta^{\frac{q}{(p-q)}}}<\frac{\varepsilon}{3}
$$

Finally, we can choose $\Delta>0$ sufficiently small for

$$
C L_{R}^{q}\left(\Delta^{\frac{q}{4}}+\frac{1}{\left|\mu^{-1}(h(\Delta))\right|^{p-q}}\right) e^{C L_{R}^{q}}<\frac{\varepsilon}{3} .
$$

This implies (4.16) as desired.

\section{Convergence rate}

Assumption 5.1. Assume that there is a pair of constants $q>2$ and $K_{1}>0$ such that

$$
\begin{aligned}
& \left(x_{1}-x_{2}\right)^{\mathrm{T}}\left(f\left(x_{1}, y_{1}\right)-f\left(x_{2}, y_{2}\right)\right)+\frac{q-1}{2}\left|g\left(x_{1}, y_{1}\right)-g\left(x_{2}, y_{2}\right)\right|^{2} \\
\leq & K_{1}\left(\left|x_{1}-x_{2}\right|^{2}+\left|y_{1}-y_{2}\right|^{2}\right)
\end{aligned}
$$

for all $x_{1}, x_{2}, y_{1}, y_{2} \in \mathbf{R}^{n}$.

Assumption 5.2. Assume that there is a pair of positive constants $r$ and $K_{2}$ such that

$$
\begin{aligned}
& \left|f\left(x_{1}, y_{1}\right)-f\left(x_{2}, y_{2}\right)\right|^{2} \vee\left|g\left(x_{1}, y_{1}\right)-g\left(x_{2}, y_{2}\right)\right|^{2} \\
\leq & K_{2}\left(1+\left|x_{1}\right|^{r}+\left|x_{2}\right|^{r}+\left|y_{1}\right|^{r}+\left|y_{2}\right|^{r}\right)\left(\left|x_{1}-x_{2}\right|^{2}+\left|y_{1}-y_{2}\right|^{2}\right)
\end{aligned}
$$

for all $x_{1}, x_{2}, y_{1}, y_{2} \in \mathbf{R}^{n}$. 
Remark 5.1. According to Assumption 5.2, we can define the function $\mu$ as

$$
\mu(R)=C R^{1+\frac{r}{2}}
$$

where the constant $C$ is related to $K_{2}$ but independent of $R$.

Theorem 5.1. Let Assumptions 2.1,2.2, 5.1 and 5.2 hold. Assume that $\bar{q} \in[2, q)$ and $q(2+r) \leq 2 p$, then, for any $\Delta \in(0,1]$, we have

$$
\mathbb{E}\left|x(T)-x_{\Delta}(T)\right|^{\bar{q}} \leq C\left(\left(\mu^{-1}(h(\Delta))\right)^{-(p-\bar{q})+\frac{\bar{q} r}{2}}+\Delta^{\frac{\bar{q}}{2}}(h(\Delta))^{\bar{q}}\right) .
$$

Proof. Let $e(t)=x(t)-x_{\Delta}(t)$ for $t \geq 0$. For each integer $R>\left|x_{0}\right|$, define the stopping time $\theta_{R}=\tau_{R} \wedge \rho_{R}$. By the Itô formula, for any $0 \leq t \leq T$, we have

$$
\begin{gathered}
\mathbb{E}\left|e\left(t \wedge \theta_{R}\right)\right|^{\bar{q}} \\
\leq \mathbb{E} \int_{0}^{t \wedge \theta_{R}} \bar{q}|e(s)|^{\bar{q}-2}\left[e^{\mathrm{T}}(s)\left(f(x(s), x([s]))-f_{\Delta}\left(\bar{x}_{\Delta}(s), \bar{x}_{\Delta}([s])\right)\right)\right. \\
\left.\quad+\frac{\bar{q}-1}{2}\left|g(x(s), x([s]))-g_{\Delta}\left(\bar{x}_{\Delta}(s), \bar{x}_{\Delta}([s])\right)\right|^{2}\right] d s \\
\leq \mathbb{E} \int_{0}^{t \wedge \theta_{R}} \bar{q}|e(s)|^{\bar{q}-2}\left[e^{\mathrm{T}}(s)\left(f(x(s), x([s]))-f\left(x_{\Delta}(s), x_{\Delta}([s])\right)\right)\right. \\
\left.+\frac{\bar{q}-1}{2}\left(1+\frac{q-\bar{q}}{\bar{q}-1}\right)\left|g(x(s), x([s]))-g\left(x_{\Delta}(s), x_{\Delta}([s])\right)\right|^{2}\right] d s \\
+\mathbb{E} \int_{0}^{t \wedge \theta_{R}} \bar{q}|e(s)|^{\bar{q}-2}\left[e^{\mathrm{T}}(s)\left(f\left(x_{\Delta}(s), x_{\Delta}([s])\right)-f_{\Delta}\left(\bar{x}_{\Delta}(s), \bar{x}_{\Delta}([s])\right)\right)\right. \\
\left.+\frac{\bar{q}-1}{2}\left(1+\frac{\bar{q}-1}{q-\bar{q}}\right)\left|g\left(x_{\Delta}(s), x_{\Delta}([s])\right)-g_{\Delta}\left(\bar{x}_{\Delta}(s), \bar{x}_{\Delta}([s])\right)\right|^{2}\right] d s .
\end{gathered}
$$

By Assumption 5.1, we have

$$
\mathbb{E}\left|e\left(t \wedge \theta_{R}\right)\right|^{\bar{q}} \leq \bar{q} K_{1} \int_{0}^{t} \sup _{0 \leq u \leq s} \mathbb{E}\left|e\left(u \wedge \theta_{R}\right)\right|^{\bar{q}} d s+J_{3},
$$

where

$$
\begin{aligned}
J_{3}=\mathbb{E} \int_{0}^{t \wedge \theta_{R}} \bar{q}|e(s)|^{\bar{q}-2}\left[e^{\mathrm{T}}(s)\left(f\left(x_{\Delta}(s), x_{\Delta}([s])\right)-f_{\Delta}\left(\bar{x}_{\Delta}(s), \bar{x}_{\Delta}([s])\right)\right)\right. \\
\left.\quad+\frac{\bar{q}-1}{2}\left(1+\frac{\bar{q}-1}{q-\bar{q}}\right)\left|g\left(x_{\Delta}(s), x_{\Delta}([s])\right)-g_{\Delta}\left(\bar{x}_{\Delta}(s), \bar{x}_{\Delta}([s])\right)\right|^{2}\right] d s .
\end{aligned}
$$

Rearranging $J_{3}$, we obtain

$$
J_{3} \leq J_{31}+J_{32},
$$


where

$$
\begin{aligned}
& J_{31}=\mathbb{E} \int_{0}^{t \wedge \theta_{R}} \bar{q}|e(s)|^{\bar{q}-2}\left[e^{\mathrm{T}}(s)\left(f\left(x_{\Delta}(s), x_{\Delta}([s])\right)-f_{\Delta}\left(x_{\Delta}(s), x_{\Delta}([s])\right)\right)\right. \\
&\left.+\frac{(\bar{q}-1)(q-1)}{q-\bar{q}}\left|g\left(x_{\Delta}(s), x_{\Delta}([s])\right)-g_{\Delta}\left(x_{\Delta}(s), x_{\Delta}([s])\right)\right|^{2}\right] d s \\
& J_{32}=\mathbb{E} \int_{0}^{t \wedge \theta_{R}} \bar{q}|e(s)|^{\bar{q}-2}\left[e^{\mathrm{T}}(s)\left(f_{\Delta}\left(x_{\Delta}(s), x_{\Delta}([s])\right)-f_{\Delta}\left(\bar{x}_{\Delta}(s), \bar{x}_{\Delta}([s])\right)\right)\right. \\
&\left.+\frac{(\bar{q}-1)(q-1)}{q-\bar{q}}\left|g_{\Delta}\left(x_{\Delta}(s), x_{\Delta}([s])\right)-g_{\Delta}\left(\bar{x}_{\Delta}(s), \bar{x}_{\Delta}([s])\right)\right|^{2}\right] d s .
\end{aligned}
$$

By the Young inequality $a^{\bar{q}-2} b \leq \frac{\bar{q}-2}{\bar{q}} a^{\bar{q}}+\frac{2}{\bar{q}} b^{\frac{\bar{q}}{2}}, \forall a, b \geq 0$, we obtain

$$
\begin{gathered}
J_{31} \leq \mathbb{E} \int_{0}^{t \wedge \theta_{R}} \bar{q}|e(s)|^{\bar{q}-2}\left[\frac{1}{2}\left|e^{\mathrm{T}}(s)\right|^{2}+\frac{1}{2}\left|f\left(x_{\Delta}(s), x_{\Delta}([s])\right)-f_{\Delta}\left(x_{\Delta}(s), x_{\Delta}([s])\right)\right|^{2}\right. \\
\left.+\frac{(\bar{q}-1)(q-1)}{q-\bar{q}}\left|g\left(x_{\Delta}(s), x_{\Delta}([s])\right)-g_{\Delta}\left(x_{\Delta}(s), x_{\Delta}([s])\right)\right|^{2}\right] d s \\
\leq \mathbb{E} \int_{0}^{t \wedge \theta_{R}}\left[\frac{(q-2)(\bar{q}-1)^{2}}{q-\bar{q}}|e(s)|^{\bar{q}}+\left|f\left(x_{\Delta}(s), x_{\Delta}([s])\right)-f_{\Delta}\left(x_{\Delta}(s), x_{\Delta}([s])\right)\right|^{\bar{q}}\right. \\
\left.+\frac{2(\bar{q}-1)(q-1)}{q-\bar{q}}\left|g_{\Delta}\left(x_{\Delta}(s), x_{\Delta}([s])\right)-g_{\Delta}\left(x_{\Delta}(s), x_{\Delta}([s])\right)\right|^{\bar{q}}\right] d s \\
\leq C \int_{0}^{t} \sup _{0 \leq u \leq s} \mathbb{E}\left|e\left(u \wedge \theta_{R}\right)\right|^{\bar{q}} d s+J_{311},
\end{gathered}
$$

where

$$
C=\max \left\{\frac{(q-2)(\bar{q}-1)^{2}}{q-\bar{q}}, 1, \frac{2(\bar{q}-1)(q-1)}{q-\bar{q}}\right\}
$$

and

$$
\begin{aligned}
J_{311}=C \mathbb{E} \int_{0}^{t \wedge \theta_{R}}\left[\left|f\left(x_{\Delta}(s), x_{\Delta}([s])\right)-f_{\Delta}\left(x_{\Delta}(s), x_{\Delta}([s])\right)\right|^{\bar{q}}\right. \\
\left.+\left|g\left(x_{\Delta}(s), x_{\Delta}([s])\right)-g_{\Delta}\left(x_{\Delta}(s), x_{\Delta}([s])\right)\right|^{\bar{q}}\right] d s .
\end{aligned}
$$

Applying Assumption 5.2, we get

$$
\begin{aligned}
J_{311} \leq 2 C K_{2} \mathbb{E} \int_{0}^{t \wedge \theta_{R}}(1+ & \left.\left|x_{\Delta}(s)\right|^{r}+\left|x_{\Delta}([s])\right|^{r}+\left|\pi_{\Delta}\left(x_{\Delta}(s)\right)\right|^{r}+\left|\pi_{\Delta}\left(x_{\Delta}([s])\right)\right|^{r}\right)^{\frac{\bar{q}}{2}} \\
& \times\left(\left|x_{\Delta}(s)-\pi_{\Delta}\left(x_{\Delta}(s)\right)\right|^{2}+\left|x_{\Delta}([s])-\pi_{\Delta}\left(x_{\Delta}([s])\right)\right|^{2}\right)^{\frac{\bar{q}}{2}} d s \\
\leq 10^{\frac{\bar{q}}{2}} C K_{2} \mathbb{E} \int_{0}^{t \wedge \theta_{R}}(1+ & \left.\left|x_{\Delta}(s)\right|^{\frac{r \bar{q}}{2}}+\left|x_{\Delta}([s])\right|^{\frac{r \bar{q}}{2}}+\left|\pi_{\Delta}\left(x_{\Delta}(s)\right)\right|^{\frac{r \bar{q}}{2}}+\left|\pi_{\Delta}\left(x_{\Delta}([s])\right)\right|^{\frac{r \bar{q}}{2}}\right) \\
& \times\left(\left|x_{\Delta}(s)-\pi_{\Delta}\left(x_{\Delta}(s)\right)\right|^{\bar{q}}+\left|x_{\Delta}([s])-\pi_{\Delta}\left(x_{\Delta}([s])\right)\right|^{\bar{q}}\right) d s
\end{aligned}
$$




$$
\begin{aligned}
\leq 10^{\frac{\bar{q}}{2}+1} C K_{2} \int_{0}^{t} \mathbb{E}(1 & \left.+\left|x_{\Delta}\left(s \wedge \theta_{R}\right)\right|^{\frac{r \bar{q}}{2}}+\left|x_{\Delta}\left(\left[s \wedge \theta_{R}\right]\right)\right|^{\frac{r \bar{q}}{2}}\right) \\
\times & \left(\left|x_{\Delta}\left(s \wedge \theta_{R}\right)-\pi_{\Delta}\left(x_{\Delta}\left(s \wedge \theta_{R}\right)\right)\right|^{\bar{q}}\right. \\
& \left.\quad+\left|x_{\Delta}\left(\left[s \wedge \theta_{R}\right]\right)-\pi_{\Delta}\left(x_{\Delta}\left(\left[s \wedge \theta_{R}\right]\right)\right)\right|^{\bar{q}}\right) d s .
\end{aligned}
$$

Applying Hölder inequality, we have

$$
\begin{aligned}
& J_{311} \leq C \int_{0}^{t}\left(\mathbb{E}\left(1+\left|x_{\Delta}\left(s \wedge \theta_{R}\right)\right|^{p}+\left|x_{\Delta}\left(\left[s \wedge \theta_{R}\right]\right)\right|^{p}\right)\right)^{\frac{\bar{q} r}{2 p}} \\
& \times\left(\mathbb { E } \left(\left|x_{\Delta}\left(s \wedge \theta_{R}\right)-\pi_{\Delta}\left(x_{\Delta}\left(s \wedge \theta_{R}\right)\right)\right|^{\frac{2 p \bar{q}}{2 p-\bar{q} r}}\right.\right. \\
& \left.\left.+\left|x_{\Delta}\left(\left[s \wedge \theta_{R}\right]\right)-\pi_{\Delta}\left(x_{\Delta}\left(\left[s \wedge \theta_{R}\right]\right)\right)\right|^{\frac{2 p \bar{q}}{2 p-\bar{q} r}}\right)\right)^{\frac{2 p-\bar{q} r}{2 p}} d s \\
& \leq C \int_{0}^{t}\left(1+\sup _{0 \leq u \leq s} \mathbb{E}\left|x_{\Delta}\left(u \wedge \theta_{R}\right)\right|^{p}\right)^{\frac{\bar{q} r}{2 p}} \\
& \times\left(\sup _{0 \leq u \leq s} \mathbb{E}\left|x_{\Delta}\left(u \wedge \theta_{R}\right)-\pi_{\Delta}\left(x_{\Delta}\left(u \wedge \theta_{R}\right)\right)\right|^{\frac{2 p \bar{q}}{2 p-\bar{q} r}}\right)^{\frac{2 p-\bar{q} r}{2 p}} d s .
\end{aligned}
$$

By Lemma 4.3 and Hölder inequality, we derive

$$
\begin{aligned}
& J_{311} \leq C \int_{0}^{t}\left(\sup _{0 \leq u \leq s} \mathbb{E}\left|x_{\Delta}\left(u \wedge \theta_{R}\right)-\pi_{\Delta}\left(x_{\Delta}\left(u \wedge \theta_{R}\right)\right)\right|^{\frac{2 p \bar{q}}{2 p-\bar{q} r}}\right)^{\frac{2 p-\bar{q} r}{2 p}} d s \\
& \leq C \int_{0}^{t}\left(\sup _{0 \leq u \leq s} \mathbb{E}\left(\left|x_{\Delta}\left(u \wedge \theta_{R}\right)\right|^{\frac{2 p \bar{q}}{2 p-\bar{q} r}} I_{\left\{\left|x_{\Delta}\left(u \wedge \theta_{R}\right)\right|>\mu^{-1}(h(\Delta))\right\}}\right)\right)^{\frac{2 p-\bar{q} r}{2 p}} d s \\
& \leq C \int_{0}^{t}\left(\sup _{0 \leq u \leq s}\left(\mathbb{E}\left|x_{\Delta}\left(u \wedge \theta_{R}\right)\right|^{p}\right)^{\frac{2 \bar{q}}{2 p-\bar{q} r}}\right. \\
& \left.\times\left(\mathbb{E}\left(I_{\left\{\left|x_{\Delta}\left(u \wedge \theta_{R}\right)\right|>\mu^{-1}(h(\Delta))\right\}}\right)^{\frac{2 p-\bar{q} r}{2(p-\bar{q})-\bar{q} r}}\right)^{\frac{2(p-\bar{q})-\bar{q} r}{2 p-\bar{q} r}}\right)^{\frac{2 p-\bar{q} r}{2 p}} d s \\
& \leq C \int_{0}^{t}\left(\sup _{0 \leq u \leq s}\left(\mathbb{P}\left\{\left|x_{\Delta}\left(u \wedge \theta_{R}\right)\right|>\mu^{-1}(h(\Delta))\right\}\right)^{\frac{2(p-\bar{q})-\bar{q} r}{2 p-\bar{q} r}}\right)^{\frac{2 p-\bar{q} r}{2 p}} d s \\
& \leq C \int_{0}^{t}\left(\sup _{0 \leq u \leq s}\left(\frac{\mathbb{E}\left|x_{\Delta}\left(u \wedge \theta_{R}\right)\right|^{p}}{\left(\mu^{-1}(h(\Delta))\right)^{p}}\right)^{\frac{2(p-\bar{q})-\bar{q} r}{2 p-\bar{q} r}}\right)^{\frac{2 p-\bar{q} r}{2 p}} d s \\
& \leq C\left(\mu^{-1}(h(\Delta))\right)^{\bar{q}\left(\frac{r}{2}+1\right)-p} \text {. }
\end{aligned}
$$


Substituting (5.15) into (5.11), we get

$$
J_{31} \leq C \int_{0}^{t} \sup _{0 \leq u \leq s} \mathbb{E}\left|e\left(u \wedge \theta_{R}\right)\right|^{\bar{q}} d s+C\left(\mu^{-1}(h(\Delta))\right)^{\bar{q}\left(\frac{r}{2}+1\right)-p} .
$$

Similarly, we can show

$$
J_{32} \leq C \int_{0}^{t} \sup _{0 \leq u \leq s} \mathbb{E}\left|e\left(u \wedge \theta_{R}\right)\right|^{\bar{q}} d s+C \Delta^{\frac{\bar{q}}{2}}(h(\Delta))^{\bar{q}} .
$$

Combining (5.6), (5.8), (5.16) and (5.17), we obtain

$$
\begin{aligned}
& \mathbb{E}\left|e\left(t \wedge \theta_{R}\right)\right|^{\bar{q}} \\
\leq & C \int_{0}^{t} \sup _{0 \leq u \leq s} \mathbb{E}\left|e\left(u \wedge \theta_{R}\right)\right|^{\bar{q}} d s+C\left(\left(\mu^{-1}(h(\Delta))\right)^{\bar{q}\left(\frac{r}{2}+1\right)-p}+\Delta^{\frac{\bar{q}}{2}}(h(\Delta))^{\bar{q}}\right) .
\end{aligned}
$$

By Gronwall inequality, we have

$$
\mathbb{E}\left|e\left(T \wedge \theta_{R}\right)\right|^{\bar{q}} \leq C\left(\left(\mu^{-1}(h(\Delta))\right)^{\bar{q}\left(\frac{r}{2}+1\right)-p}+\Delta^{\frac{\bar{q}}{2}}(h(\Delta))^{\bar{q}}\right) .
$$

Using the well-known Fatou lemma, the required assertion (5.4) follows from $R \rightarrow$ $\infty$.

Remark 5.2. (1) Assumption 5.1 implies Assumption 2.2 for any $p>2$. In fact,

$$
\begin{aligned}
& x^{\mathrm{T}} f(x, y)+\frac{p-1}{2}|g(x, y)|^{2} \\
\leq & x^{\mathrm{T}}(f(x, y)-f(0,0))+x^{\mathrm{T}} f(0,0) \\
& +\frac{p-1}{2}|g(x, y)-g(0,0)|^{2}+\frac{p-1}{2}|g(0,0)|^{2} \\
\leq & K_{1}\left(|x|^{2}+|y|^{2}\right)+\frac{1}{2}|x|^{2}+\frac{1}{2}|f(0,0)|^{2}+\frac{p-1}{2}|g(0,0)|^{2} \\
\leq & K\left(1+|x|^{2}+|y|^{2}\right),
\end{aligned}
$$

where

$$
K=\max \left\{K_{1}+\frac{1}{2}, \frac{1}{2}|f(0,0)|^{2}+\frac{p-1}{2}|g(0,0)|^{2}\right\} .
$$

(2) We may define

$$
h(\Delta)=\Delta^{-\varepsilon}
$$

for some $\varepsilon \in\left(0, \frac{1}{4}\right]$. Hence, (5.4) can be rewritten as

$$
\begin{aligned}
& \mathbb{E}\left|x(T)-x_{\Delta}(T)\right|^{\bar{q}} \\
\leq & C\left(\Delta^{\frac{\varepsilon[2(p-\bar{q})-\bar{q} r]}{2+r}}+\Delta^{\bar{q}\left(\frac{1}{2}-\varepsilon\right)}\right) \leq C \Delta\left(\frac{(2(p-\bar{q})-\bar{q} r)}{2+r} \wedge\left(\bar{q}\left(\frac{1}{2}-\varepsilon\right)\right)\right) .
\end{aligned}
$$


If we choose $p$ sufficiently large for

$$
\frac{\varepsilon(2(p-\bar{q})-\bar{q} r)}{2+r} \geq \bar{q}\left(\frac{1}{2}-\varepsilon\right)
$$

we can get

$$
\mathbb{E}\left|x(T)-x_{\Delta}(T)\right|^{\bar{q}} \leq C \Delta^{\bar{q}\left(\frac{1}{2}-\varepsilon\right)} .
$$

This remark shows that the order of convergence could be close to $\frac{1}{2}$ arbitrarily.

\section{Stability analysis}

In this section, we set $f(0,0)=0$ and $g(0,0)=0$. By Assumption 2.1, we have

$$
\sup _{|x| \vee|y| \leq r}|f(x, y)| \leq 2 r L_{r}
$$

In the following, we define

$$
\mu(r)=2 r L_{r},
$$

where $L_{r}$ is the local Lipschitz coefficient in Assumption 2.1. This operation links the local Lipschitz coefficients $L_{r}$ to the truncation function $\mu(r)$, ensuring that the upper bound of the step size can be finally obtained, which is clear and easy to verify.

Definition 6.1. The SDEPCAs (2.1) is said to be exponentially stable in mean square if there exists a pair of positive constants $\lambda$ and $M$ such that for any initial value $x_{0}$

$$
\mathbb{E}|x(t)|^{2} \leq M\left|x_{0}\right|^{2} e^{-\lambda t}, \quad \forall t>0,
$$

where $\lambda$ is the rate constant and $M$ is the growth constant.

Definition 6.2. For a given step size $\Delta>0$, the truncated Euler-Maruyama method is said to be exponentially stable in mean square if there exists a pair of positive constants $\gamma$ and $H$ such that for any initial value $x_{0}$

$$
\mathbb{E}\left|X_{\Delta}\left(t_{s m+l}\right)\right|^{2} \leq H\left|x_{0}\right|^{2} e^{-\gamma(s m+l) \Delta}
$$

for all $s \in \mathbb{N}, l=0, \ldots, m-1$, where $\gamma$ is the rate constant and $H$ is the growth constant.

Assumption 6.1. Assume that there are positive constants $\lambda_{1}>\lambda_{2}>0$, such that $f$ and $g$ satisfy

$$
x^{T} f(x, y)+\frac{1}{2}|g(x, y)|^{2} \leq-\lambda_{1}|x|^{2}+\lambda_{2}|y|^{2}, \quad \forall x, y \in \mathbf{R}^{n} .
$$

Under Assumption 6.1, the exponential stability of SDEPCAs (2.1) has been obtained in [13]. 
Lemma 6.1. Assume that Assumption 6.1 holds. Then, for all $\Delta \in(0,1)$, we have

$$
x^{T} f_{\Delta}(x, y)+\frac{1}{2}\left|g_{\Delta}(x, y)\right|^{2} \leq\left(-\lambda_{1}+\frac{\lambda_{2}}{2}\right)|x|^{2}+\frac{\lambda_{2}}{2}|y|^{2}, \quad \forall x, y \in \boldsymbol{R}^{n},
$$

where $\lambda_{1}$ and $\lambda_{2}$ are the same as Assumption 6.1.

The proof is similar to Lemma 3.1, so we omit it. In the following, we firstly state two conclusions obtained in [13] as lemmas for the use of this paper.

Lemma 6.2. Let $z_{s m+l}$ be a sequence of numbers, $s \in \mathbb{N}, l=0, \ldots, m-1$. If there are constants $\alpha>\beta>0$ such that $1-\alpha \Delta>0$ and

$$
z_{s m+l+1} \leq(1-\alpha \Delta) z_{s m+l}+\beta \Delta z_{s m}
$$

then

$$
z_{s m+l+1} \leq\left(\frac{\beta}{\alpha}+\left(1-\frac{\beta}{\alpha}\right) e^{-\alpha(l+1) \Delta}\right) z_{s m} .
$$

Lemma 6.3. Assume that $\alpha, \beta$ are two positive constants. If $\alpha>\beta$, then for all $t>0$ we have

$$
0<\frac{\beta}{\alpha}+\left(1-\frac{\beta}{\alpha}\right) e^{-\alpha t}<1
$$

For simplicity of presentation, we set

$$
\begin{aligned}
& \alpha=2 \lambda_{1}-\beta, \quad \beta=2 L_{\mu^{-1}(h(\Delta))}^{2} \Delta+\lambda_{2}, \\
& r(l+1)=\frac{\beta}{\alpha}+\left(1-\frac{\beta}{\alpha}\right) e^{-\alpha(l+1) \Delta},
\end{aligned}
$$

where $l=0, \ldots, m-1$. The main theorem of this section is given below.

Theorem 6.1. Let Assumptions 2.1 and 6.1 hold. Then for every $0<\Delta<\Delta^{*}=$ $\min \left\{\frac{1}{2 \lambda_{1}}, \frac{4\left(\lambda_{1}-\lambda_{2}\right)^{2}}{\hat{h}^{4}}\right\}$ and any initial value $x_{0} \in \boldsymbol{R}^{n}$, the truncated Euler-Maruyama method (3.6) is exponentially stable in mean square with rate constant $\gamma=-\log r(m)$ and growth constant $H=\frac{1}{r(m)}$. have

Proof. By Assumption 2.1, Lemma 6.1 and the property of Brownian motion, we

$$
\begin{aligned}
& \mathbb{E}\left|X_{\Delta}\left(t_{s m+l+1}\right)\right|^{2} \\
\leq & \mathbb{E}\left|X_{\Delta}\left(t_{s m+l}\right)\right|^{2}+2 L_{\mu^{-1}(h(\Delta))}^{2} \Delta^{2}\left(\mathbb{E}\left|X_{\Delta}\left(t_{s m+l}\right)\right|^{2}+\mathbb{E}\left|X_{\Delta}\left(t_{s m}\right)\right|^{2}\right) \\
& +2 \Delta\left(-\lambda_{1}+\frac{\lambda_{2}}{2}\right) \mathbb{E}\left|X_{\Delta}\left(t_{s m+l}\right)\right|^{2}+\lambda_{2} \Delta \mathbb{E}\left|X_{\Delta}\left(t_{s m}\right)\right|^{2} \\
= & (1-\alpha \Delta) \mathbb{E}\left|X_{\Delta}\left(t_{s m+l}\right)\right|^{2}+\beta \Delta \mathbb{E}\left|X_{\Delta}\left(t_{s m}\right)\right|^{2} .
\end{aligned}
$$


By (6.2), we have

$$
L_{\mu^{-1}(h(\Delta))}=\frac{h(\Delta)}{2 \mu^{-1}(h(\Delta))}
$$

Recalling that $h(\Delta)>\mu(1)$, we have $\mu^{-1}(h(\Delta))>1$. Applying (3.2) and $\Delta<\frac{4\left(\lambda_{1}-\lambda_{2}\right)^{2}}{\hat{h}^{4}}$, we obtain

$$
\beta=2\left(\frac{h(\Delta)}{2 \mu^{-1}(h(\Delta))}\right)^{2} \Delta+\lambda_{2}<\lambda_{1} .
$$

Hence $\alpha>\beta>0$. By $\Delta<\frac{1}{2 \lambda_{1}}$, we have

$$
\alpha \Delta=\left(2 \lambda_{1}-\beta\right) \Delta<\frac{2 \lambda_{1}-\beta}{2 \lambda_{1}}<1 .
$$

Applying Lemma 6.2, we have

$$
\begin{aligned}
\mathbb{E}\left|X_{\Delta}\left(t_{s m+l+1}\right)\right|^{2} & \leq(1-\alpha \Delta) \mathbb{E}\left|X_{\Delta}\left(t_{s m+l}\right)\right|^{2}+\beta \Delta \mathbb{E}\left|X_{\Delta}\left(t_{s m}\right)\right|^{2} \\
& \leq\left(\frac{\beta}{\alpha}+\left(1-\frac{\beta}{\alpha}\right) e^{-\alpha(l+1) \Delta}\right) \mathbb{E}\left|X_{\Delta}\left(t_{s m}\right)\right|^{2} \\
& =r(l+1) \mathbb{E}\left|X_{\Delta}\left(t_{s m}\right)\right|^{2} .
\end{aligned}
$$

By Lemma 6.3, we have $0<r(l+1)<1$. If $l=m-1$, the above equation can be written as

$$
\mathbb{E}\left|X_{\Delta}\left(t_{(s+1) m}\right)\right|^{2} \leq r(m) \mathbb{E}\left|X_{\Delta}\left(t_{s m}\right)\right|^{2} \leq \cdots \leq(r(m))^{s+1}\left|x_{0}\right|^{2} .
$$

Submitting (6.15) into (6.14), we can obtain

$$
\begin{aligned}
& \mathbb{E}\left|X_{\Delta}\left(t_{s m+l+1}\right)\right|^{2} \\
\leq & r(l+1) r(m)^{s}\left|x_{0}\right|^{2}=\frac{r(l+1)}{r(m)^{(l+1) \Delta}}\left|x_{0}\right|^{2} e^{(s m+l+1) \Delta \log (r(m))} \\
\leq & \frac{1}{r(m)} e^{(s m+l+1) \Delta \log (r(m))}\left|x_{0}\right|^{2}=H e^{-\gamma(s m+l+1) \Delta}\left|x_{0}\right|^{2},
\end{aligned}
$$

where $H=\frac{1}{r(m)}$ and $\gamma=-\log (r(m))>0$.

\section{Numerical simulation}

This section gives some examples to illustrate the results above. The convergence theory is tested by the first two examples. The third example verifies the stability.

Example 7.1. We consider the following scalar SDEPCAs

$$
d x(t)=\left(-x^{3}(t)+x([t])\right) d t+\left(|x(t)|^{\frac{3}{2}}+x([t])\right) d B(t)
$$

with initial value $x(0)=1, t \in[0,2]$. 


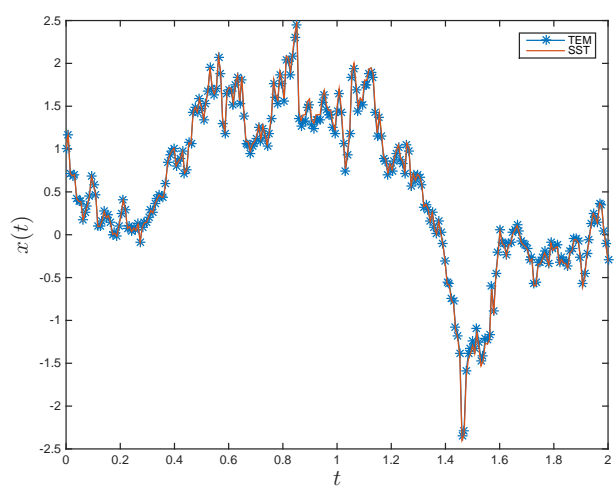

Figure 1: One path of the exact solution and the numerical solution for one dimension.

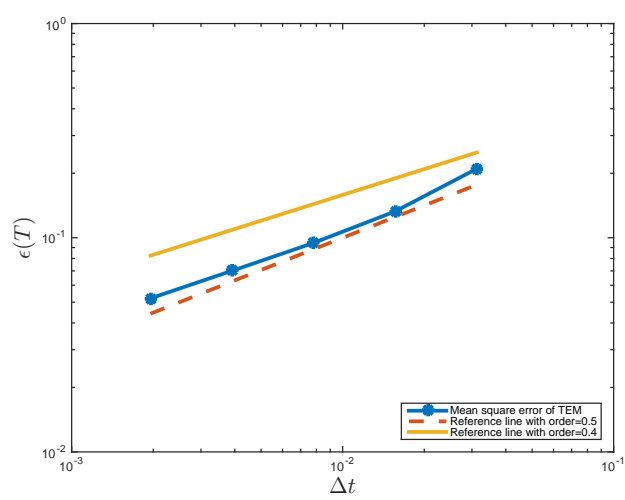

Figure 2: Convergence order at $T=2$.

Let $\Delta=\frac{1}{m}$, then the split-step theta (SST) method for (7.1) is as follows.

$$
\begin{aligned}
& \hat{x}_{s m+l}=x_{s m+l}+\theta \Delta\left(-\hat{x}_{s m+l}^{3}+x_{s m}\right) \\
& x_{s m+l+1}=x_{s m+l}+\Delta\left(-\hat{x}_{s m+l}^{3}+x_{s m}\right)+\left(\left|\hat{x}_{s m+l}\right|^{\frac{3}{2}}+x_{s m}\right) \Delta B_{s m+l} .
\end{aligned}
$$

According to [14], the SST method with $\theta \in\left[\frac{1}{2}, 1\right]$ is strongly convergent to SDEPCAs. We use the SST method with step size $\Delta=2^{-14}$ and $\theta=1$ as the "exact solution". The coefficients of (7.1) satisfy Assumptions 2.1, 2.2, 5.1 and 5.2 with $q=3, \bar{q}=2, r=4$ and $p=30$. Moreover, let $\mu(r)=2 r^{3}$ and $h(\Delta)=\left(\frac{125}{4}\right) \Delta^{-\frac{1}{10}}$. Fig. 1 shows that the truncated EM method is convergent to the exact solution. We take 100 samples and choose $\Delta=2^{-8}$. Table 1 shows the number of truncation applied in each sample. We also compute the numerical solutions using different step sizes $\Delta=2^{-9}, 2^{-8}, 2^{-7}, 2^{-6}, 2^{-5}$ on the same Brownian path and obtain corresponding errors. We take $M=5000$.

Let $\epsilon$ denote the error in mean square, then by the law of large numbers, at the final

Table 1: The number of truncation applied in each sample.

\begin{tabular}{||llllllllll||}
512 & 448 & 512 & 509 & 512 & 508 & 507 & 496 & 512 & 499 \\
490 & 512 & 490 & 507 & 431 & 499 & 493 & 511 & 512 & 512 \\
465 & 511 & 455 & 512 & 475 & 512 & 510 & 506 & 508 & 512 \\
499 & 492 & 512 & 512 & 504 & 462 & 511 & 512 & 510 & 485 \\
512 & 397 & 459 & 480 & 507 & 512 & 499 & 489 & 475 & 482 \\
477 & 512 & 512 & 512 & 500 & 512 & 486 & 504 & 512 & 512 \\
512 & 487 & 512 & 499 & 491 & 512 & 512 & 512 & 512 & 509 \\
511 & 498 & 509 & 512 & 512 & 507 & 511 & 512 & 493 & 495 \\
510 & 486 & 512 & 489 & 512 & 509 & 511 & 512 & 502 & 512 \\
512 & 492 & 511 & 511 & 509 & 512 & 512 & 512 & 512 & 508 \\
\hline
\end{tabular}


Table 2: The Monte Carlo errors for (7.1).

\begin{tabular}{||c|ccccc||}
\hline$\Delta t$ & 1000 & 2000 & 3000 & 4000 & 5000 \\
\hline $2^{-9}$ & 0.00310153 & 0.00248716 & 0.00193821 & 0.00160219 & 0.00144283 \\
$2^{-8}$ & 0.00380181 & 0.00312375 & 0.00244000 & 0.00205428 & 0.00193796 \\
$2^{-7}$ & 0.00504908 & 0.00403356 & 0.00322514 & 0.00274647 & 0.00261020 \\
$2^{-6}$ & 0.00783951 & 0.00554820 & 0.00464228 & 0.00391444 & 0.00364825 \\
$2^{-5}$ & 0.01303230 & 0.00953383 & 0.00758430 & 0.00639328 & 0.00568892 \\
\hline
\end{tabular}

integer time $T, \epsilon$ satisfies

$$
\epsilon(T)=\left(\mathbb{E}\left|x(T)-X_{\Delta}(T)\right|^{2}\right)^{\frac{1}{2}} \approx\left(\frac{1}{M} \sum_{i=1}^{M}\left|x\left(T, w_{i}\right)-X_{\Delta}\left(T, w_{i}\right)\right|^{2}\right)^{\frac{1}{2}} .
$$

The log-log plot of the strong errors against the step sizes is shown in Fig. 2. Compared with the reference line with order 0.5 , it can be verified that the order of the strong error is close to 0.5 .

The Monte Carlo errors with confidence level $1-\alpha$ is defined as follows.

$$
\varepsilon=\frac{\lambda_{\alpha} \sigma}{\sqrt{M}}
$$

where $\lambda_{\alpha}$ and $\alpha$ are one-to-one correspondence, $\sigma$ is the standard deviation of $\mid x(T)-$ $\left.X_{\Delta}(T)\right|^{2}$. Since $\sigma$ can not be obtained exactly, we use the estimation $\hat{\sigma}$.

$$
\hat{\sigma}=\sqrt{\frac{1}{M} \sum_{i=1}^{M}\left|x\left(T, w_{i}\right)-X_{\Delta}\left(T, w_{i}\right)\right|^{4}-\left(\frac{1}{M} \sum_{i=1}^{M}\left|x\left(T, w_{i}\right)-X_{\Delta}\left(T, w_{i}\right)\right|^{2}\right)^{2}} .
$$

Table 2 shows the Monte Carlo error with $\alpha=0.05$ and $\lambda_{\alpha}=1.96$.

Example 7.2. Consider the following two dimensional SDEPCA:

$$
\left\{\begin{array}{l}
d x_{1}(t)=\left(-x_{1}(t)-6 x_{1}^{3}(t)\right) d t+\left|x_{2}(t)\right|^{\frac{3}{2}} d B(t) \\
d x_{2}(t)=\left(-x_{2}(t)-6 x_{2}^{3}(t)+x_{1}^{2}(t)\right) d t+\left|x_{1}([t])\right| d B(t)
\end{array}\right.
$$

with initial data $x_{1}(0)=1.5$ and $x_{2}(0)=1$.

$$
\begin{aligned}
& \text { Let } x(t)=\left(x_{1}(t), x_{2}(t)\right)^{T}, y(t)=\left(x_{1}([t]), x_{2}([t])\right)^{T} \text { and } \\
& f_{1}(x, y)=-x_{1}-6 x_{1}^{3}, \quad f_{2}(x, y)=-x_{2}-6 x_{2}^{3}+x_{1}^{2}, \\
& g_{1}(x, y)=\left|x_{2}\right|^{\frac{3}{2}}, \quad g_{2}(x, y)=\left|y_{1}\right|, \\
& f(x, y)=\left(f_{1}(x, y), f_{2}(x, y)\right)^{T}, \quad g(x, y)=\left(g_{1}(x, y), g_{2}(x, y)\right)^{T} \text {. }
\end{aligned}
$$




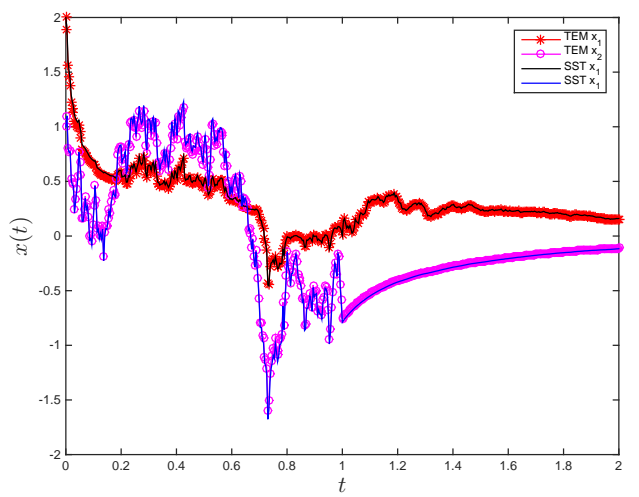

Figure 3: One path of the exact solution and the numerical solution for multi dimension.

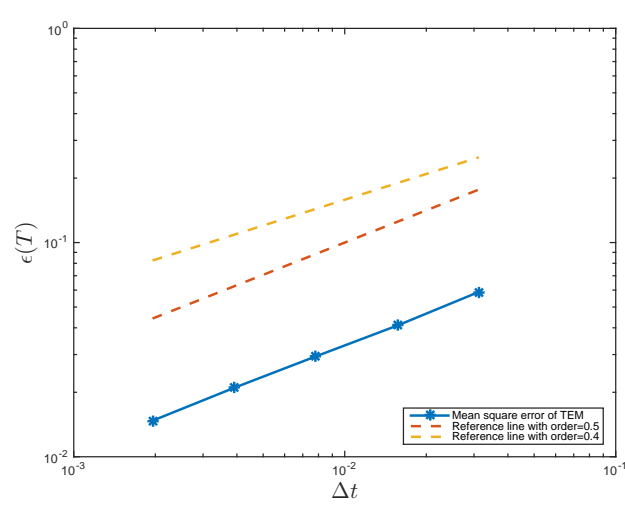

Figure 4: Convergence order at $T=2$.

The coefficients of (7.7) satisfy Assumptions 2.1, 2.2, 5.1 and 5.2. Clearly,

$$
\sup _{|x| \vee|y| \leq r}(|f(x, y)| \vee|g(x, y)|) \leq 14 r^{3}, \quad \forall r \geq 1 .
$$

Then we choose $\mu(r)=14 r^{3}$. Moreover, choose $h(\Delta)=56 \Delta^{-\frac{1}{10}}$. We use the split-step theta method with step size $\Delta=2^{-14}$ as the "exact solution". We also compute the numerical solutions using different step sizes $\Delta=2^{-9}, 2^{-8}, 2^{-7}, 2^{-6}, 2^{-5}$ on the same Brownian path and obtain the corresponding errors. We take 5000 sample paths. Fig. 3 shows that the truncated EM method converges to the exact solution. The log-log plot of the strong errors against the step sizes is shown in Fig. 4. Table 3 shows the Monte Carlo error with confidence level 0.95 .

Table 3: The Monte Carlo errors for (7.7).

\begin{tabular}{||c|ccccc||}
\hline$\Delta t$ & 1000 & 2000 & 3000 & 4000 & 5000 \\
\hline $2^{-9}$ & 0.00003079 & 0.00002650 & 0.00002014 & 0.00001752 & 0.00001544 \\
$2^{-8}$ & 0.00007564 & 0.00007700 & 0.00005478 & 0.00004534 & 0.00003861 \\
$2^{-7}$ & 0.00010589 & 0.00010254 & 0.00008480 & 0.00007772 & 0.00006680 \\
$2^{-6}$ & 0.00015667 & 0.00016543 & 0.00020037 & 0.00016448 & 0.00014073 \\
$2^{-5}$ & 0.00046301 & 0.00032523 & 0.00027995 & 0.00024947 & 0.00022006 \\
\hline
\end{tabular}

Example 7.3. Consider the following SDEPCAs

$$
d x(t)=\left(-x^{3}(t) x^{2}([t])-x(t)\right) d t+\left(x^{2}(t) x([t])+0.4 x([t])\right) d B(t)
$$

with initial data $x(0)=8$.

The coefficients satisfy both Assumptions 2.1 and 6.1 with $\lambda_{1}=1, \lambda_{2}=0.16$. We test the stability of the truncated Euler-Maruyama method with three different 


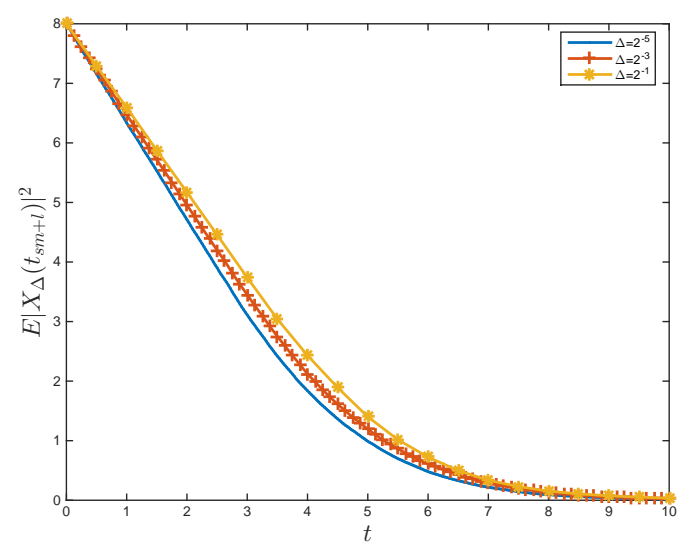

Figure 5: The mean square exponential stability of the numerical solutions with different step sizes.

step sizes $\Delta=2^{-5}, 2^{-3}$ and $2^{-1}$. We take 5000 sample paths. The truncated EulerMaruyama method is stable if $\Delta<\frac{1}{2}$. The mean square of the numerical solutions is plotted in Fig. 5.

\section{Conclusions}

In this paper, we have analyzed the convergence and stability of the truncated EulerMaruyama method for SDEPCAs. We obtain that the convergence rate is closed to 0.5 arbitrarily without any restrictions for the step size. Moreover, we have shown that the truncated Euler-Maruyama method preserves the exponential mean square stability of the SDEPCAs under some restrictions on the step size.

\section{Acknowledgments}

The authors thank the referees and the editor for their valuable comments and helpful suggestions. This work is supported by the National Natural Science Foundation of China (No. 11671113) and the National Postdoctoral Program for Innovative Talents (No. BX20180347).

\section{References}

[1] K. DAREIOTIS, C. KumAR, AND S. SABANiS, On tamed Euler approximations of SDEs driven by Lévy noise with applications to delay equations, SIAM J. Numer. Anal., 54 (2016), 18401872.

[2] J. GAO, S. MA, AND H. LIANG, Strong convergence of the semi-implicit Euler method for a kind of stochastic Volterra integro-differential equations, Numer. Math. Theory Methods Appl., 12 (2019), 547-565. 
[3] Q. GuO, W. LiU, X. MAO, AND R. Yue, The partially truncated Euler-Maruyama method and its stability and boundedness, Appl. Numer. Math., 115 (2017), 235-251.

[4] D. J. Higham, X. MAO, AND A. M. STUART, Strong convergence of Euler-type methods for nonlinear stochastic differential equations, SIAM J. Numer. Anal., 40 (2002), 1041-1063.

[5] L. Hu, X. LI, AND X. MAO, Convergence rate and stability of the truncated Euler-Maruyama method for stochastic differential equations, J. Comput. Appl. Math., 337 (2018), 274-289.

[6] Y. Hu, Semi-implicit Euler-Maruyama scheme for stiff stochastic equations, in Stochastic Analysis and Related Topics V, Springer, 1996, 183-202.

[7] M. Hutzenthaler, A. Jentzen, And P. E. Kloeden, Strong and weak divergence in finite time of Euler's method for stochastic differential equations with non-globally Lipschitz continuous coefficients, Proc. R. Soc. Lond. Ser. A Math. Phys. Eng. Sci., 467 (2011), 15631576.

[8] M. Hutzenthaler, A. Jentzen, And P. E. Kloeden, Strong convergence of an explicit numerical method for SDEs with nonglobally Lipschitz continuous coefficients, Ann. Appl. Probab., 22 (2012), 1611-1641.

[9] T. KÜPPER AND R. YUAN, On quasi-periodic solutions of differential equations with piecewise constant argument, J. Math. Anal. Appl., 267 (2002), 173-193.

[10] G. LAN AND F. XIA, Strong convergence rates of modified truncated EM method for stochastic differential equations, J. Comput. Appl. Math., 334 (2018), 1-17.

[11] X. LI, Existence and exponential stability of solutions for stochastic cellular neural networks with piecewise constant argument, J. Appl. Math., (2014).

[12] W. LIU AND X. MAO, Strong convergence of the stopped Euler-Maruyama method for nonlinear stochastic differential equations, Appl. Math. Comput., 223 (2013), 389-400.

[13] Y. Lu, M. SONG, AND M. LIU, Convergence and stability of the split-step theta method for stochastic differential equations with piecewise continuous arguments, J. Comput. Appl. Math., 317 (2017), 55-71.

[14] Y. Lu, M. SONG, AND M. LIU, Convergence rate and stability of the split-step theta method for stochastic differential equations with piecewise continuous arguments, Discrete Contin. Dyn. Syst. Ser. B 24, (2019).

[15] X. MAO, Stochastic differential equations and applications, Horwood, Chichester, 2007.

[16] X. MAO, The truncated Euler-Maruyama method for stochastic differential equations, J. Comput. Appl. Math., 290 (2015), 370-384.

[17] X. MAO, Almost sure exponential stabilization by discrete-time stochastic feedback control, IEEE Trans. Automat. Control, 61 (2016), 1619-1624.

[18] X. MAO, Convergence rates of the truncated Euler-Maruyama method for stochastic differential equations, J. Comput. Appl. Math., 296 (2016), 362-375.

[19] X. MAO AND L. SZPRUCH, Strong convergence rates for backward Euler-Maruyama method for non-linear dissipative-type stochastic differential equations with super-linear diffusion coefficients, Stochastics, 85 (2013), 144-171.

[20] M. MilošEvić, The Euler-Maruyama approximation of solutions to stochastic differential equations with piecewise constant arguments, J. Comput. Appl. Math., 298 (2016), 1-12.

[21] A. D. MYshKis, On certain problems in the theory of differential equations with deviating argument, Russian Math. Surveys, 32 (1977), 181-213.

[22] R. QI AND Q. LIN, Time-stepping error bound for a stochastic parabolic Volterra equation disturbed by fractional Brownian motions, Numer. Math. Theory Methods Appl., 12 (2019), 778-796.

[23] Q. QIU, W. LIU, L. Hu, X. MAO, AND S. You, Stabilization of stochastic differential equations with Markovian switching by feedback control based on discrete-time state observation 
with a time delay, Statist. Probab. Lett., 115 (2016), 16-26.

[24] M. SonG, Y. Lu, AND M. LIU, Convergence of the tamed Euler method for stochastic differential equations with piecewise continuous arguments under non-global Lipschitz continuous coefficients, Numer. Funct. Anal. Optim., 39 (2018), 517-536.

[25] H. SU, X. MAO, AND W. LI, Hopf bifurcation control for a class of delay differential systems with discrete-time delayed feedback controller, Chaos, 26(11) (2016), 1-20.

[26] M. V. TRETYAKov AND Z. Zhang, A fundamental mean-square convergence theorem for SDEs with locally Lipschitz coefficients and its applications, SIAM J. Numer. Anal., 51 (2013), 3135-3162.

[27] J. Wiener, Generalized solutions of functional differential equations, World Scientific, 1993.

[28] S. You, W. LiU, J. Lu, X. MAO, AND Q. QIU, Stabilization of hybrid systems by feedback control based on discrete-time state observations, SIAM J. Control Optim., 53 (2015), 905925. 